\title{
Facets of the generalized Cluster complex and regions in the extended Catalan arrangement of type $A$
}

\author{
Susanna Fishel* \\ School of Mathematical and Statistical Sciences \\ Arizona State University \\ Tempe, AZ 85287, U.S.A. \\ fishel@math.asu.edu
}

\author{
Myrto Kallipoliti ${ }^{\dagger}$ \\ Fakultatät für Mathematik \\ Universität Wien \\ 1090 Vienna, Austria \\ myrto.kallipoliti@univie.ac.at
}

\author{
Eleni Tzanaki \\ Department of Applied Mathematics \\ University of Crete \\ 71409 Heraklion, Crete, Greece \\ etzanaki@tem.uoc.gr
}

Submitted: Mar 1, 2013; Accepted: Oct 10, 2013; Published: Oct 21, 2013

Mathematics Subject Classifications: 05A17, 05E45, 52C35

\begin{abstract}
In this paper we present a bijection between two well known families of Catalan objects: the set of facets of the $m$-generalized cluster complex $\Delta^{m}\left(A_{n}\right)$ and that of dominant regions in the $m$-Catalan arrangement Cat $^{m}\left(A_{n}\right)$, where $m \in \mathbb{N}_{>0}$. In particular, the map which we define bijects facets containing the negative simple root $-\alpha$ to dominant regions having the hyperplane $\{v \in V \mid\langle v, \alpha\rangle=m\}$ as separating wall. As a result, it restricts to a bijection between the set of facets of the positive part of $\Delta^{m}\left(A_{n}\right)$ and the set of bounded dominant regions in Cat ${ }^{m}\left(A_{n}\right)$. Our map is a composition of two bijections in which integer partitions in an $m$-dilated $n$-staircase shape come into play.
\end{abstract}

\section{Introduction}

Fomin and Zelevinsky defined the cluster complex $\Delta(\Phi)$, a pure simplicial complex associated to every finite root system $\Phi[12]$, as a tool for the study of finite cluster algebras

${ }^{*}$ Partially supported by Simons Foundation grant no. 209806 and NSF grant no. 1200280.

${ }^{\dagger}$ Supported by FWF research grant no. Z130-N13. 
[11]. The ground set of $\Delta(\Phi)$ is the set of almost positive roots, $\Phi_{\geqslant-1}$, which consists of all positive roots and all negative simple roots. Fomin and Reading [10] generalized it to the $m$-cluster complex $\Delta^{m}(\Phi)$, a pure simplicial complex defined for any finite (possibly reducible) Coxeter group and nonnegative integer $m$. The ground set of $\Delta^{m}(\Phi)$ is the set of colored almost positive roots, $\Phi_{\geqslant-1}^{m}$, which consists of one copy of each negative simple root together with $m$ copies of each positive root. When $m=1$, the complex $\Delta^{m}(\Phi)$ reduces to $\Delta(\Phi)$. The generalized cluster complex contains a natural subcomplex, called the positive part of $\Delta^{m}(\Phi)$ and denoted by $\Delta_{+}^{m}(\Phi)$. It consists of faces of $\Delta^{m}(\Phi)$ that do not contain negative simple roots.

When $\Phi$ is irreducible, the number of facets of $\Delta^{m}(\Phi)$ and $\Delta_{+}^{m}(\Phi)$ is equal to the $m$ Catalan number $N(\Phi, m)=\prod_{i=1}^{n} \frac{e_{i}+m h+1}{e_{i}+1}$ and the positive $m$-Catalan number $N_{+}(\Phi, m)=$ $\prod_{i=1}^{n} \frac{e_{i}+m h-1}{e_{i}+1}$ respectively, where $n$ is the rank, $h$ is the Coxeter number and $e_{i}$ are the exponents of $\Phi$. In the special case where $\Phi=A_{n-1}$, we obtain the well-known FussCatalan numbers

$$
N(\Phi, m)=\frac{1}{m n+1}\left(\begin{array}{c}
(m+1) n \\
n
\end{array}\right) .
$$

The Fuss-Catalan numbers count an enormous number of combinatorial objects. For instance, they count the number of $m$-Dyck (or $m$-ballot) paths of size $n$. These are lattice paths from $(0,0)$ to $(m n, n)$ using north steps $(0,1)$ and east steps $(1,0)$ which do not go below the line $y=\frac{1}{m} x$. Equivalently, they count the number of integer partitions whose Young diagram is contained in the $m$-staircase shape defined by the partition $(m(n-1), m(n-2), \ldots, m)$. In this paper we use such integer partitions as a way to encode the facets of the generalized cluster complex $\Delta^{m}\left(A_{n-1}\right)$, as well as the dominant regions of the extended Catalan arrangement of type $A_{n-1}$.

The dominant regions of the Catalan arrangement $\operatorname{Cat}^{m}\left(A_{n}\right)$ constitute the second object of our study. In this introduction we give a brief description of the hyperplane arrangement $\mathrm{Cat}^{m}(\Phi)$ in the general setting of any finite crystallographic root system $\Phi$ and we refer the reader to [2-4] for more details. In the proofs, however, we focus only in the special case where $\Phi=A_{n}$. Let $\Phi$ be a finite crystallographic root system with set of positive roots $\Phi_{>0}$ and let $V$ be the Euclidean space spanned by the set $\Phi_{>0}$ with inner product $\langle\cdot, \cdot\rangle$. The $m$-Catalan arrangement $\mathrm{Cat}^{m}(\Phi)$ is the collection of hyperplanes $\left\{H_{\alpha, k} \mid \alpha \in \Phi_{>0}, 0 \leqslant k \leqslant m\right\}$, where $H_{\alpha, k}=\{v \in V \mid\langle v, \alpha\rangle=k\}$ for $\alpha \in \Phi$ and $k \in \mathbb{Z}$. The hyperplanes of $\mathrm{Cat}^{m}(\Phi)$ dissect $V$ into regions. The dominant chamber of $V$ is the intersection $\bigcap_{\alpha \in \Phi_{>0}}\{v \in V \mid\langle v, \alpha\rangle \geqslant 0\}$, and is also referred to as the fundamental chamber in the literature. Every region contained in the dominant chamber is called a dominant region. Note that the dominant regions in the $m$-Catalan arrangement are the same as the dominant regions in the $m$-Shi arrangement $[24,26]$. The bounded regions in $\operatorname{Cat}^{m}(\Phi)$ are those which are bounded in the usual topological sense. It is proved in $[2$, Lemma 4.1] that a dominant region $R$ is bounded if and only if $0<\langle\mathbf{x}, \alpha\rangle<m$ for all $\mathbf{x} \in R$ and all simple roots $\alpha$ in $\Phi$. When $\Phi$ is an irreducible crystallographic root system, the number of dominant and bounded dominant regions in $\operatorname{Cat}^{m}(\Phi)$ is again $N(\Phi, m)$ and $N_{+}(\Phi, m)$ respectively.

Summarizing, for every finite crystallographic root system we have the same number of 
facets of $\Delta^{m}(\Phi)$ as we have dominant regions in $\mathrm{Cat}^{m}(\Phi)$. Moreover, the number of facets of the positive cluster complex $\Delta_{+}^{m}(\Phi)$ is equal to the number of bounded dominant regions in the $m$-Catalan arrangement $\mathrm{Cat}^{m}(\Phi)$. We seek a bijection to explain these coincidences. For $m=1$ there exist bijections between non-crossing partitions and cluster complexes [20], as well as between non-crossing partitions and dominant regions in $\operatorname{Cat}^{1}(\Phi)[1,7,17$. However, for $m \geqslant 2$ few results that relate the above objects are known $[6,16,27]$. To the best of our knowledge, there is no known bijection between dominant regions in $\mathrm{Cat}^{m}(\Phi)$ and facets of the cluster complex $\Delta^{m}(\Phi)$.

In this paper we close this gap in the case where $\Phi=A_{n}$. Before we continue, we need to fix some notation. Let $\mathcal{D}^{m}\left(A_{n}\right)$ (or $\mathcal{D}_{n}^{m}$ for short) be the set of facets of the $m$-generalized cluster complex $\Delta^{m}\left(A_{n}\right)$ and let $\mathcal{R}^{m}\left(A_{n}\right)$ (or $\mathcal{R}_{n}^{m}$ for short) be the set of dominant regions in the $m$-Catalan arrangement $\operatorname{Cat}^{m}\left(A_{n}\right)$. We recall that a wall of a region $R$ is a hyperplane in $\operatorname{Cat}^{m}(\Phi)$ which supports a facet of $R$. We say that $H$ is a separating wall of $R$, if the region $R$ and the origin lie in different half-spaces relative to $H$. Finally, for $n \in \mathbb{N}$ we set $[n]=\{1,2, \ldots, n\}$ and $[n]_{0}=\{0,1, \ldots, n\}$. The main result of this paper is the following bijection.

Bijection 1. Let $\left\{\alpha_{1}, \alpha_{2}, \ldots, \alpha_{n}\right\}$ be the set of simple roots of type $A_{n}$. We construct a bijection $\omega: \mathcal{D}_{n}^{m} \rightarrow \mathcal{R}_{n}^{m}$ with the property that, for any $i \in[n]$, the negative simple root $-\alpha_{i}$ is contained in the facet $D \in \mathcal{D}_{n}^{m}$ if and only if the hyperplane $H_{\alpha_{i}, m}$ is a separating wall of the region $\omega(D)$.

We remark that the map $\omega$ of Bijection 1 restricts to one between facets of $\Delta_{+}^{m}\left(A_{n}\right)$ and bounded regions in $\mathrm{Cat}^{m}\left(A_{n}\right)$. Even further, $\omega$ satisfies a certain refinement of this; it gives a combinatorial proof of the fact that for any $J \subseteq[n]$, the number of facets of $\Delta^{m}\left(A_{n}\right)$ containing exactly the negative simple roots $-\alpha_{i}$ with $i \in J$, is equal to the number of dominant regions in $\operatorname{Cat}^{m}\left(A_{n}\right)$ with simple separating walls $H_{\alpha_{i}, m}$ with $i \in J$, where $\left\{\alpha_{1}, \alpha_{2}, \ldots, \alpha_{n}\right\}$ is the set of simple roots of type $A_{n}$.

In order to construct the bijection $\omega$, we associate dominant regions of the $m$-Catalan arrangement $\operatorname{Cat}^{m}\left(A_{n}\right)$ to facets of the $m$-generalized cluster complex $\Delta^{m}\left(A_{n}\right)$ via certain integer partitions. More precisely, as mentioned above, we use as intermediaries integer partitions whose Young diagram fits inside the diagram of the partition $(m n, m(n-$ $1), \ldots, m)$. We denote the set of such partitions by $\mathcal{P}^{m}\left(A_{n}\right)$ (or $\mathcal{P}_{n}^{m}$ for short). Partitions in $\mathcal{P}_{n}^{m}$ can also be viewed as lattice paths from $(0,0)$ to $(m n, n)$ using north and east steps and stay above the line $y=\frac{1}{m} x-1$, or equivalently $m$-Dyck, or $m$-ballot paths of size $n+1$. Our main idea is to biject both, facets of the cluster complex and dominant regions of $\operatorname{Cat}^{m}\left(A_{n}\right)$, to partitions in $\mathcal{P}_{n}^{m}$. A facet containing the negative simple root $-\alpha_{i}$ becomes a partition $\left(\lambda_{1}, \lambda_{2}, \ldots, \lambda_{n}\right)$ whose $i$-th part has maximum size, that is $\lambda_{i}=m(n-i+1)$. On the other hand, a dominant region with simple separating wall $H_{\alpha_{i}, m}$ also bijects to a partition whose $i$-th part has maximum size. We remark that a maximum-sized part of a partition in $\mathcal{P}_{n}^{m}$ corresponds to a point where the lattice path touches a line of the form $y=\frac{x}{m}-1$, or where the $m$-Dyck path touches the line $y=\frac{1}{m} x$. Touch points (also called contacts) appear often in the literature. For instance, in [5], touch points are used to derive a recursion to count the number of intervals in a generalization of the $m$-Tamari 
lattice and in [14] sorting 1-Dyck paths by touch points leads to a refinement of the shuffle conjecture. In this paper we give two bijections, which eventually lead to the construction of $\omega$.

Bijection 2. Let $\left\{\alpha_{1}, \alpha_{2}, \ldots, \alpha_{n}\right\}$ be the set of simple roots of type $A_{n}$. We construct a bijection $\varphi: \mathcal{R}_{n}^{m} \rightarrow \mathcal{P}_{n}^{m}$ with the property that, for any $i \in[n]$, the hyperplane $H_{\alpha_{i}, m}$ is a separating wall of the region $R \in \mathcal{R}_{n}^{m}$ if and only if the $i$-th part of the partition $\varphi(R)$ is equal to $m(n-i+1)$.

Bijection 3. Let $\left\{\alpha_{1}, \alpha_{2}, \ldots, \alpha_{n}\right\}$ be the set of simple roots of type $A_{n}$. We construct a bijection $\psi: \mathcal{D}_{n}^{m} \rightarrow \mathcal{P}_{n}^{m}$ with the property that, for any $i \in[n]$, the negative simple root $-\alpha_{i}$ is contained in the facet $D \in \mathcal{D}_{n}^{m}$ if and only if the $i$-th part of the partition $\psi(D)$ is equal to $m(n-i+1)$.

This paper is structured as follows. In Section 2 we fix notation and provide the necessary background. We note that throughout this work we use a realization of the facets of the cluster complex in terms of polygon dissections given by S. Fomin and N. Reading [10]. We also realize the dominant regions in terms of certain tableaux which are called Shi tableaux. Since these realizations will be crucial for our proofs, in Sections 2.2 and 2.42.5, respectively, we describe them in detail. In Sections 3 and 4 we provide respectively Bijections 2 and 3. Their composition is the desired map $\omega$ of Bijection 1. We conclude with Section 5, where we explain our future directions and state an open question.

\section{Preliminaries}

\subsection{Root system of type $A_{n}$}

Let $\varepsilon_{1}, \varepsilon_{2}, \ldots, \varepsilon_{n+1}$ be the standard basis in $\mathbb{R}^{n+1}$. In type $A_{n}$, a standard choice of positive and simple roots are respectively, the sets $\left\{\alpha_{i j} \mid 1 \leqslant i \leqslant j \leqslant n\right\}$ and $\left\{\alpha_{i} \mid 1 \leqslant i \leqslant n\right\}$, where $\alpha_{i j}:=\varepsilon_{i}-\varepsilon_{j+1}$ and $\alpha_{i}:=\varepsilon_{i}-\varepsilon_{i+1}$, for every $1 \leqslant i \leqslant j \leqslant n$. The positive roots can be written in terms of the simple roots as

$$
\alpha_{i j}=\alpha_{i}+\alpha_{i+1}+\cdots+\alpha_{j} \text {, for every } 1 \leqslant i \leqslant j \leqslant n .
$$

For notational consistency, we will often write $\alpha_{i, i}$ instead of $\alpha_{i}$.

\subsection{Cluster complexes and polygon dissections}

The $m$-generalized cluster complex $\Delta^{m}(\Phi)$ can be defined for any finite root system $\Phi$ of rank $n$. More precisely, $\Delta^{m}(\Phi)$ is a pure simplicial complex of dimension $n-1$ on the ground set of colored almost positive roots i.e the set of $m$ "colored" copies of each positive root and one copy of each negative simple root. An important result of [10] is that, in the special case where $\Phi$ is a finite classical root system, $\Delta^{m}(\Phi)$ can be realized in terms of polygon dissections. 
In what follows we describe the above mentioned realization in the case where $\Phi=A_{n}$ and we refer the reader to [10, Section 5.1] for more details. Let $P$ be a polygon with $m(n+1)+2$ vertices. An $m$-diagonal in $P$ is a diagonal dissecting $P$ into a pair of polygons, each of which has number of vertices equal to 2 modulo $m$. Notice that a collection of $n$ such $m$-diagonals is an $(m+2)$-angulation of $P$. Let $P$ be a polygon with $m(n+1)+2$ vertices labeled from 0 up to $m(n+1)+1$ in counterclockwise order. For $1 \leqslant i \leqslant \frac{n+1}{2}$, the negative simple root $-\alpha_{2 i-1}$ is identified with the diagonal of $P$ connecting the vertex $(i-1) m$ to the vertex $(n+1-i) m+1$. For $1 \leqslant i \leqslant \frac{n}{2}$, we identify the negative simple root $-\alpha_{2 i}$ with the diagonal of $P$ connecting the vertex $i m$ to the vertex $(n+1-i) m+1$. Notice that the negative simple roots form a "snake" in $P$ (see Figure $1(\alpha)$ ). For each pair $(i, j)$ with $1 \leqslant i \leqslant j \leqslant n+1$ there are exactly $n$-many $m$-diagonals that intersect (in their interior) the diagonals $-\alpha_{i},-\alpha_{i+1}, \ldots,-\alpha_{j}$ and no other diagonals in the snake. The diagonal corresponding to the colored positive root $\alpha_{i j}^{k}$ is uniquely determined as the $k$-th in clockwise order of the exactly $n m$-diagonals which intersect $-\alpha_{i},-\alpha_{i+1}, \ldots,-\alpha_{j}$ (see Figure $1(\beta)$ ). Thus, every $m$-diagonal in $P$ corresponds to a colored almost positive root in $\Delta^{m}\left(A_{n}\right)$. Under this identification, the faces of $\Delta^{m}\left(A_{n}\right)$ of dimension $k$ are in bijection with dissections of $P$ having $k+1$ pairwise non crossing $m$-diagonals. The facets of $\Delta^{m}\left(A_{n}\right)$ are dissections with $n$-many such diagonals or equivalently $(m+2)$-angulations of $P$.

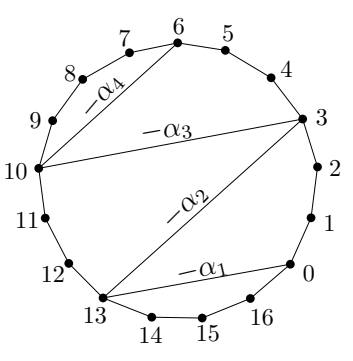

$(\alpha)$

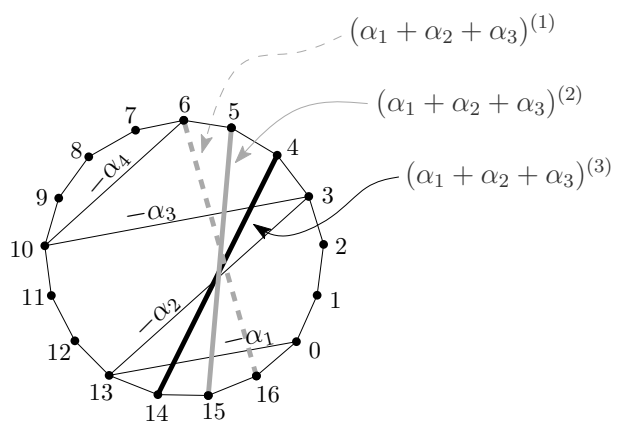

$(\beta)$

Figure 1: $(\alpha)$ the "snake" formed by the negative simple roots in $\Delta^{3}\left(A_{4}\right) .(\beta)$ the three colored copies of the positive root $\alpha_{13}=\alpha_{1}+\alpha_{2}+\alpha_{3}$.

We denote by $\mathcal{D}^{m}\left(A_{n}\right)$ (or $\mathcal{D}_{n}^{m}$ for short) the set of $(m+2)$-angulations of an $(m(n+$ $1)+2$ )-gon $P$ and, for any $i \in[n]$, we say that $D \in \mathcal{D}_{n}^{m}$ contains the negative simple root $-\alpha_{i}$ if the diagonal corresponding to $-\alpha_{i}$ is contained in $D$.

\subsection{Integer partitions}

An integer partition is a nonincreasing sequence $\lambda=\left(\lambda_{1}, \lambda_{2}, \ldots, \lambda_{n}\right)$ of nonnegative integers, called parts. We identify a partition $\lambda=\left(\lambda_{1}, \lambda_{2}, \ldots, \lambda_{n}\right)$ with its Young diagram, the left-justified array of boxes with $\lambda_{i}$ boxes in row $i$. The box in row $i$, column $j$, has 
coordinates $(i, j)$. In what follows we describe the partitions we are interested in. Let $n$ and $m$ be positive integers. We denote by $\mathcal{P}^{m}\left(A_{n}\right)$ (or $\mathcal{P}_{n}^{m}$ for short) the set of partitions whose Young diagram fits into an $m$-dilated staircase shape of size $n$ (see Figure 2 ), that is:

$$
\mathcal{P}_{n}^{m}=\left\{\left(\lambda_{1}, \lambda_{2}, \ldots, \lambda_{n}\right) \mid 0 \leqslant \lambda_{i} \leqslant m(n-i+1) \text {, for every } i \in[n]\right\}
$$

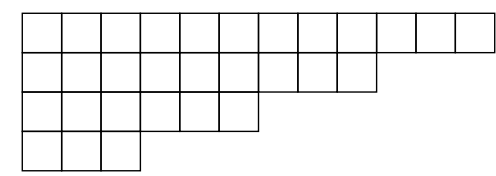

Figure 2: A 3-dilated staircase shape of size 4.

It is well known that the number of partitions in $\mathcal{P}_{n}^{m}$ is the Catalan number $N\left(A_{n}, m\right)=\frac{1}{m(n+1)+1}\left(\begin{array}{c}(m+1)(n+1) \\ n+1\end{array}\right)$. Consider now the subset $\tilde{\mathcal{P}}_{n}^{m}$ of $\mathcal{P}_{n}^{m}$ having partitions with $\lambda_{i}<m(n-i+1)$ for all $1 \leqslant i \leqslant n$. These partitions can be viewed as paths from $(0,0)$ to $(m n, n)$ which never touch the line $y=\frac{1}{m} x-1$, or equivalently, as paths from $(0,0)$ to $(n, m(n+1))$ which never touch the line $y=m x$ after the point $(0,0)$. From [13, Theorem 3] one can deduce that there are $\frac{1}{n+1}\left(\begin{array}{c}m(n+1)+n-1 \\ n\end{array}\right)$ such paths. Since $N^{+}\left(A_{n}, m\right)=\frac{1}{n+1}\left(\begin{array}{c}m(n+1)+n-1 \\ n\end{array}\right)$ (direct calculations) it is reasonable to require that our maps biject partitions of $\tilde{\mathcal{P}}_{n}^{m}$ to facets of $\Delta_{+}^{m}\left(A_{n}\right)$ as well as to bounded dominant regions in $\operatorname{Cat}^{m}\left(A_{n}\right)$. This is indeed the case, as one can see from Corollaries 3.3 and 4.5.

\subsection{Dominant regions}

Let $A_{n}^{+}$denote the set of positive roots of type $A_{n}$ and $V$ the Euclidean space spanned by $A_{n}^{+}$. For $k \in[m]_{0}$, we consider the collection of hyperplane arrangements $\mathcal{H}_{k}:=$

$\bigcup_{\alpha \in A_{n}^{+}}\{v \in V \mid\langle v, \alpha\rangle=k\}$. The dominant regions in the $m$-Catalan arrangement $\mathrm{Cat}^{m}\left(A_{n}\right)$ are precisely the connected components of the hyperplane arrangement complement $V \backslash \bigcup_{k=0}^{m} \mathcal{H}_{k}$, which lie in the dominant chamber $\bigcap_{\alpha \in A_{n}^{+}}\{v \in V \mid\langle v, \alpha\rangle \geqslant 0\}$.

Based on an idea of Shi, who arranges the positive roots in diagrams [23], we assign to each dominant region coordinates which describe its location in the Catalan arrangement. To achieve this, we remark that each dominant region $R$ in $\operatorname{Cat}^{m}\left(A_{n}\right)$ is in fact defined by a set of inequalities as follows: for every $1 \leqslant i \leqslant j \leqslant n$ and $\mathbf{x} \in R$, either

(i) there exists an integer $k_{\alpha_{i, j}} \in[m-1]_{0}$ such that $k_{\alpha_{i, j}}<\left\langle\alpha_{i j}, \mathbf{x}\right\rangle<k_{\alpha_{i, j}}+1$, or

(ii) $m<\left(\alpha_{i j}, \mathbf{x}\right)$.

We set $k_{i, j}:=k_{\alpha_{i, j}}$ in the first case and $k_{i, j}=m$ in the second one. This observation leads to the following definition. 
Definition 2.1. Let $R$ be a region in $\mathcal{R}_{n}^{m}$ and consider the integers $k_{i, j}$ defined as above. The integers $k_{i, j}$ are called coordinates of $R$, and the staircase Young diagram of size $(n, n-1, \ldots, 1)$ where $k_{i, j}$ is the entry of the box $(i, n-j+1)$, is called the Shi tableau of $R$.

Figure 3 illustrates each dominant region in $\operatorname{Cat}^{3}\left(A_{2}\right)$ together with its Shi tableau. Figure $4(\alpha)$ shows the distribution of the coordinates of a Shi tableau in the case where $\Phi=A_{4}$.

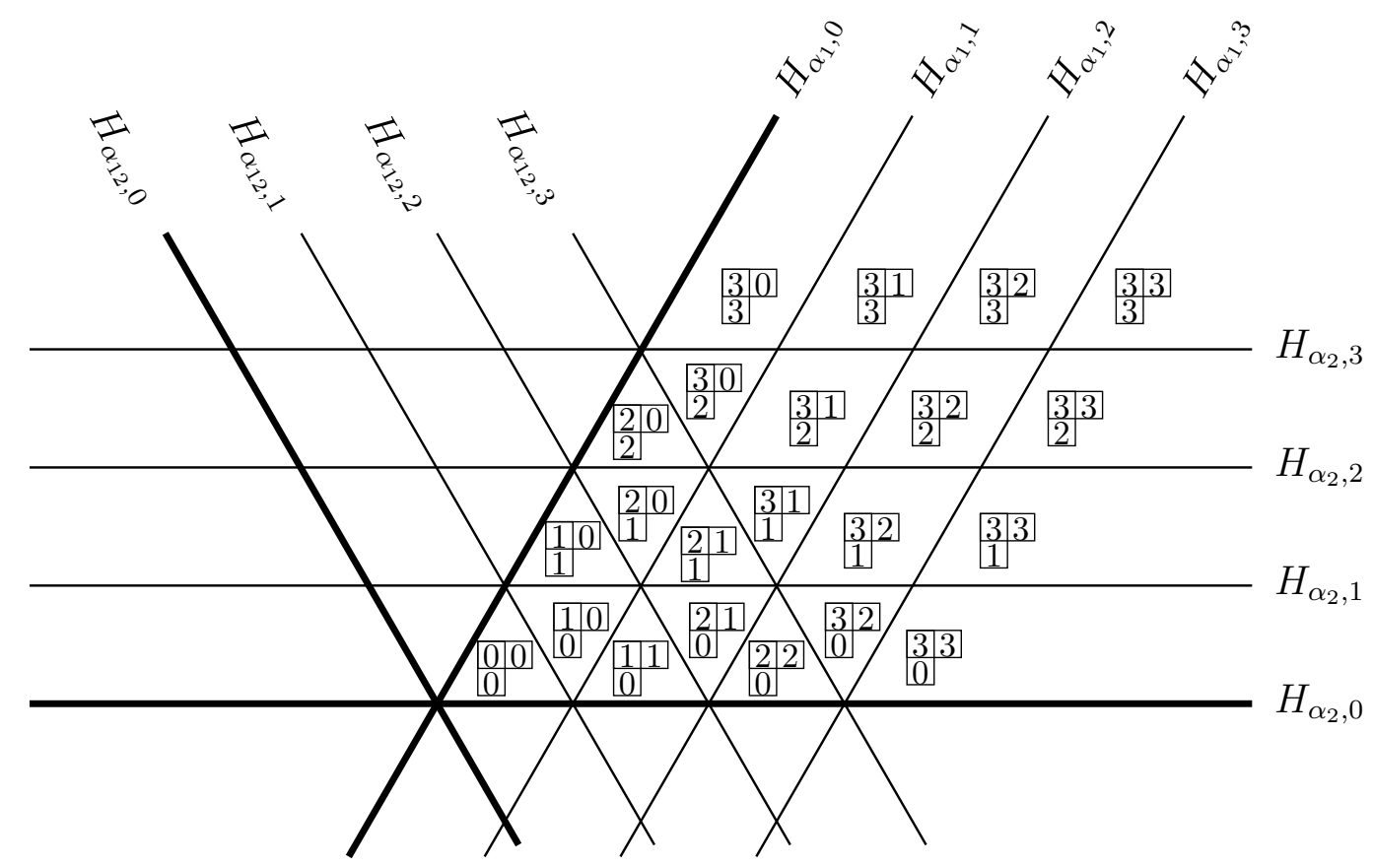

Figure 3: The hyperplane arrangement $\operatorname{Cat}^{3}\left(A_{2}\right)$ together with the Shi tableau of each dominant region.

\subsection{Shi conditions}

The entries of a Shi tableau satisfy certain conditions [3]. In order to describe them explicitly, we need to fix some notation. Let $\mathrm{x}$ be a box in a Young diagram T. A hook $\mathrm{h}$ on a box $\mathrm{x}$ of $T$ is an array of contiguous boxes lying to the right (and same row) or below (and same column) of $\mathrm{x}$ including the box $\mathrm{x}$ itself. We say that the hook $\mathrm{h}$ has length $\ell$ if the number of such boxes is equal to $\ell$. The box $\mathrm{x}$ is called the corner of the hook. If $\mathrm{h}$ is a hook of a Young diagram whose boxes are filled with numbers, we denote by $\mathrm{e}(\mathrm{h})$ the sum of the numbers at the endpoints $h$. The following characterization for the entries of a Shi tableau can be deduced from results in $[3,22]$. A more detailed proof can be found in [9, Lemma 2.5]. 
Proposition 2.2. Let $T=\left\{k_{i, j} \mid 1 \leqslant i \leqslant j \leqslant n\right\}$ be a set of integers in $[m]_{0}$ arranged in a staircase diagram of size $(n, n-1, \ldots, 1)$ so that $k_{i, j}$ is the entry of the box $(i, n-j+1)$. The set $T$ is a Shi tableau of a region $R \in \mathcal{R}_{n}^{m}$ if and only if for every $1 \leqslant i<j \leqslant n$ and any hook $\mathrm{h}_{k_{i, j}}$ of length $j-i+2$ on $k_{i, j}$ we have

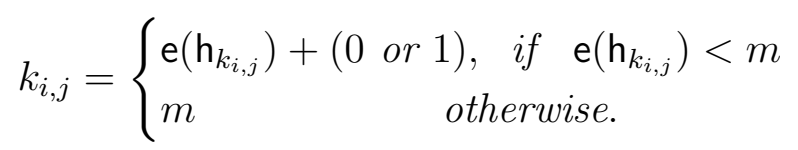

Equivalently, $T$ is a Shi tableau of a region $R \in \mathcal{R}_{n}^{m}$, if and only if for every $1 \leqslant i<j \leqslant n$ and all $i \leqslant \ell<j$ we have

$$
k_{i, j}= \begin{cases}k_{i, \ell}+k_{\ell+1, j}+\delta_{i, \ell}, & \text { if } k_{i, \ell}+k_{\ell+1, j}<m \\ m & \text { otherwise }\end{cases}
$$

where $\delta_{i, \ell} \in\{0,1\}$.

The conditions in (2.1) (or equivalently in (2.2)) holding for the entries of a Shi tableau $T$, are referred to as the Shi conditions on $T$. To check that a staircase Young diagram with numbered boxes is a Shi tableau, one has to verify the Shi conditions on each entry of the diagram. For instance, Figure 4 illustrates how the Shi conditions for a fixed choice of $k_{i, j}$ are checked. A direct consequence of the Shi conditions is that the entries of a Shi tableau $T$ (weakly) decrease along columns and rows.

Proposition 2.2 allows us to view each dominant region $R \in \mathcal{R}_{n}^{m}$ and its Shi tableau $T$ as the same object. Thus, in the remainder of the paper we do not distinguish between regions in $\mathcal{R}_{n}^{m}$ and their Shi tableaux.

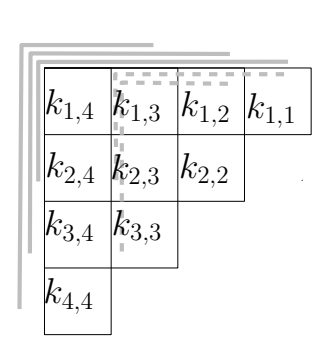

$(\alpha)$

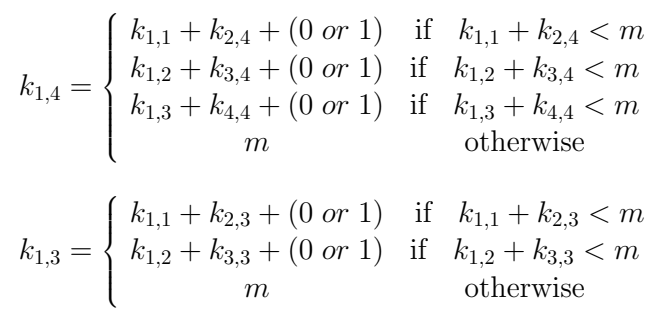

$(\beta)$

Figure 4: $(\alpha)$ The distribution of the coordinates in a 4-staircase diagram for the root system $A_{4}$. ( $\left.\beta\right)$ The gray and dashed gray hooks indicate the pairs of coordinates one should sum up to check the Shi conditions for $k_{1,4}$ and $k_{1,3}$ respectively.

\section{From dominant regions of $\mathcal{R}_{n}^{m}$ to partitions in $\mathcal{P}_{n}^{m}$}

In this section we present Bijection 2. More precisely, in Theorem 3.1 we provide a bijection $\varphi$ between the set $\mathcal{R}_{n}^{m}$ of dominant regions and the set of partitions in $\mathcal{P}_{n}^{m}$, and in 
Theorem 3.2 we prove that this map has the desired property, namely that for any simple root $\alpha_{i}$ of type $A_{n}$, the dominant regions in $\mathcal{R}_{n}^{m}$ having simple separating wall $H_{\alpha_{i}, m}$ biject to the partitions in $\mathcal{P}_{n}^{m}$ whose $i$-th part is maximal. Before we proceed with the proofs, a few comments on the history of this bijection are in order. In [25,26], Stanley defined a bijection between regions in the extended Shi arrangement and generalized parking functions. In both papers, the bijection is recursively constructed. When restricted to the dominant chamber, the parking functions in the image can be seen as partitions and ours agrees with it. Richards [21] explicitly defined a bijection from dominant regions to partitions and again, ours agrees with it. He proved it was an injection and used the sizes of the sets involved to prove it was a bijection. Although our result agrees with the above mentioned ones, our contribution lies in the fact that we encode the regions by their Shi tableaux, which makes the statement of the bijection much more concrete. As already mentioned, we do not distinguish between regions in $\mathcal{R}_{n}^{m}$ and their Shi tableaux.

Theorem 3.1. The map $\varphi: \mathcal{R}_{n}^{m} \rightarrow \mathcal{P}_{n}^{m}$, which sends each Shi tableau $R \in \mathcal{R}_{n}^{m}$ to the partition $\Lambda=\left(\lambda_{1}, \lambda_{2}, \ldots, \lambda_{n}\right)$ with $\lambda_{i}=\sum_{j=i}^{n} k_{i, j}$, is a bijection with inverse map $\varphi^{-1}(\Lambda)=$ $R_{\Lambda}$, where $R_{\Lambda}$ is the Shi tableau (defined recursively) with entries:

$$
k_{i, j}(\Lambda)=\min \left\{m,\left\lceil\frac{\lambda_{i}-\sum_{\ell=j+1}^{n} k_{i, \ell}(\Lambda)+\sum_{\ell=i}^{j-1} k_{\ell+1, j}(\Lambda)}{j-i+1}\right]\right\}, \quad 1 \leqslant i \leqslant j \leqslant n .
$$

Proof. Let $R=\left\{k_{i, j}: 1 \leqslant i \leqslant j \leqslant n\right\}$ be a Shi tableau in $\mathcal{R}_{n}^{m}$ and set $\lambda_{i}=\sum_{j=i}^{n} k_{i, j}$. Since the entries of a Shi tableau decrease along columns, the image $\varphi(R)=\Lambda$ of $R$ is a partition. Moreover, since all $k_{i, j} \leqslant m$, we conclude that $\Lambda \in \mathcal{P}_{n}^{m}$. In order to show that $\varphi$ is an injection, we will define its inverse map. To this end, we consider some $\Lambda=\left(\lambda_{1}, \lambda_{2}, \ldots, \lambda_{n}\right) \in \mathcal{P}_{n}^{m}$ and we want to define integers $k_{i, j}(\Lambda) \in[m]_{0}$ so that:

(i) $\left\{k_{i, j}(\Lambda): 1 \leqslant i \leqslant j \leqslant n\right\} \in \mathcal{R}_{n}^{m}$ (i.e. all $k_{i, j}(\Lambda)$ satisfy the Shi conditions), and

(ii) $\lambda_{i}=\sum_{j=i}^{n} k_{i, j}(\Lambda)$ for every $i \in[n]$.

To do this, we assume that (i) and (ii) hold and we try to express each such $k_{i, j}(\Lambda)$ in terms of parts of $\Lambda$ and those $k_{i, j}$ 's lying below and to its left. We separate two cases, which both lead to expression (3.1). In what follows, for simplicity, we write $k_{i, j}$ instead of $k_{i, j}(\Lambda)$.

Case 1: Let $k_{i, j}<m$. In this case, the Shi conditions for $k_{i, j}$ can be written as:

$$
\left.\begin{array}{rl}
k_{i, j}= & k_{i, i}+k_{i+1, j}+\delta_{i, i} \\
k_{i, j}= & k_{i, i+1}+k_{i+2, j}+\delta_{i, i+1} \\
& \vdots \\
k_{i, j}= & k_{i, j-1}+k_{j, j}+\delta_{i, j-1} \\
k_{i, j}= & k_{i, j}
\end{array}\right\}
$$


where $\delta_{i, \ell} \in\{0,1\}$ for all $i \leqslant \ell<j$.

Summing up the above equalities, we get

$$
\begin{aligned}
(j-i+1) k_{i, j} & =\sum_{\ell=i}^{j} k_{i, \ell}+\sum_{\ell=i}^{j-1} k_{\ell+1, j}+\sum_{\ell=i}^{j-1} \delta_{i, \ell} \\
& =\sum_{\ell=i}^{n} k_{i, \ell}-\sum_{\ell=j+1}^{n} k_{i, \ell}+\sum_{\ell=i}^{j-1} k_{\ell+1, j}+\sum_{\ell=i}^{j-1} \delta_{i, \ell} \\
& =\lambda_{i}-\sum_{\ell=j+1}^{n} k_{i, \ell}+\sum_{\ell=i}^{j-1} k_{\ell+1, j}+\Delta,
\end{aligned}
$$

where $\Delta$ is an integer with $0 \leqslant \Delta \leqslant j-i$. Thus,

$$
\begin{aligned}
& k_{i, j}=\frac{\lambda_{i}-\sum_{\ell=j+1}^{n} k_{i, \ell}+\sum_{\ell=i}^{j-1} k_{\ell+1, j}}{j-i+1}+\frac{\Delta}{j-i+1} \\
& \text { or } \quad k_{i, j}-\frac{\lambda_{i}-\sum_{\ell=j+1}^{n} k_{i, \ell}+\sum_{\ell=i}^{j-1} k_{\ell+1, j}}{j-i+1}=\frac{\Delta}{j-i+1} \\
& \text { or }\left\lfloor k_{i, j}-\frac{\lambda_{i}-\sum_{\ell=j+1}^{n} k_{i, \ell}+\sum_{\ell=i}^{j-1} k_{\ell+1, j}}{j-i+1}\right\rfloor=\left\lfloor\frac{\Delta}{j-i+1}\right\rfloor .
\end{aligned}
$$

Since $k_{i, j}$ is an integer and $0 \leqslant \frac{\Delta}{j-i+1}<1$ we have

$$
\begin{aligned}
k_{i, j}+ & \left\lfloor-\frac{\lambda_{i}-\sum_{\ell=j+1}^{n} k_{i, \ell}+\sum_{\ell=i}^{j-1} k_{\ell+1, j}}{j-i+1}\right\rfloor=0 \\
\text { or } \quad k_{i, j}- & \left\lceil\frac{\lambda_{i}-\sum_{\ell=j+1}^{n} k_{i, \ell}+\sum_{\ell=i}^{j-1} k_{\ell+1, j}}{j-i+1}\right\rceil=0 \\
\text { or } \quad k_{i, j}= & =\left[\frac{\lambda_{i}-\sum_{\ell=j+1}^{n} k_{i, \ell}+\sum_{\ell=i}^{j-1} k_{\ell+1, j}}{j-i+1}\right] .
\end{aligned}
$$

Under the assumption that $k_{i, j}<m$, we can write

$$
k_{i, j}=\min \left\{m,\left\lceil\frac{\lambda_{i}-\sum_{\ell=j+1}^{n} k_{i, \ell}+\sum_{\ell=i}^{j-1} k_{\ell+1, j}}{j-i+1}\right\rceil\right\},
$$

which is indeed the expression (3.1) in the statement of Theorem 3.1.

Case 2: Let $k_{i, j}=m$. To show that $k_{i, j}=\min \left\{m,\left\lceil\frac{\lambda_{i}-\sum_{\ell=j+1}^{n} k_{i, \ell}+\sum_{\ell=i}^{j-1} k_{\ell+1, j}}{j-i+1}\right\rceil\right\}$ it suffices to prove that

$$
\left\lceil\frac{\lambda_{i}-\sum_{\ell=j+1}^{n} k_{i, \ell}+\sum_{\ell=i}^{j-1} k_{\ell+1, j}}{j-i+1}\right\rceil \geqslant m
$$


To this end, notice than when $k_{i, j}=m$ the Shi conditions imply that $k_{i, \ell}+k_{\ell+1, j} \geqslant m-1$ for every $\ell$ with $i \leqslant \ell<j$. We thus have

$$
\begin{aligned}
\frac{\lambda_{i}-\sum_{\ell=j+1}^{n} k_{i, \ell}+\sum_{\ell=i}^{j-1} k_{\ell+1, j}}{j-i+1} & =\frac{\sum_{\ell=i}^{j} k_{i, \ell}+\sum_{\ell=i}^{j-1} k_{\ell+1, j}}{j-i+1} \\
& =\frac{k_{i, j}+\sum_{\ell=i}^{j-1} k_{i, \ell}+\sum_{\ell=i}^{j-1} k_{\ell+1, j}}{j-i+1} \\
& \geqslant \frac{m+(j-i)(m-1)}{j-i+1} \\
& >\frac{m-1+(j-i)(m-1)}{j-i+1}=m-1
\end{aligned}
$$

Using the inequality $\lceil x\rceil \geqslant x$ for all $x \in \mathbb{R}$, the above implies

$$
\left\lceil\frac{\lambda_{i}-\sum_{\ell=j+1}^{n} k_{i, \ell}+\sum_{\ell=i+1}^{j} k_{\ell, j}}{j-i+1}\right\rceil>m-1
$$

and since both, left and right hand side of the above inequality, are integers we can equivalently write

$$
\left\lceil\frac{\lambda_{i}-\sum_{\ell=j+1}^{n} k_{i, \ell}+\sum_{\ell=i+1}^{j} k_{\ell, j}}{j-i+1}\right\rceil \geqslant m,
$$

as we wanted.

Finally, since the sets $\mathcal{P}_{n}^{m}$ and $\mathcal{R}_{n}^{m}$ have the same cardinality, we conclude that our injection is a bijection. This completes our proof.

Theorem 3.2. Let $\left\{\alpha_{1}, \alpha_{2}, \ldots, \alpha_{n}\right\}$ be the set of simple roots of type $A_{n}$. The map $\varphi: \mathcal{R}_{n}^{m} \rightarrow \mathcal{P}_{n}^{m}$ of Theorem 3.1 has the property that for every $i \in[n]$, the hyperplane $H_{\alpha_{i}, m}$ is a separating wall of $R \in \mathcal{R}_{n}^{m}$ if and only if the $i$-th part of $\varphi(R)$ is maximal, that is $\lambda_{i}=m(n-i+1)$.

Proof. First, let $R$ be a region in $\mathcal{R}_{n}^{m}$ with simple separating wall $H_{\alpha_{i}, m}$ for some $i \in[n]$. This implies that $\left\langle\alpha_{i}, \mathbf{x}\right\rangle>m$ for all $\mathbf{x} \in R$ which, in view of Definition 2.1, means that $k_{i, i}=m$. Since the entries of the Shi tableau decrease along rows we deduce that $k_{i, \ell} \geqslant k_{i, i}=m$ for all $i \leqslant \ell \leqslant n$. This, along with the fact that all entries $k_{i, j} \leqslant m$, implies that $k_{i, \ell}=m$ for all $i \leqslant \ell \leqslant n$. Thus, the $i$-th part $\lambda_{i}=\sum_{\ell=i}^{n} k_{i, \ell}$ of $\varphi(R)$ is indeed $m(n-i+1)$.

Before we proceed with the reverse direction of the proof, a remark is in order. Let $R$ be a region in $\mathcal{R}_{n}^{m}$ and $\alpha$ a positive root in $\Phi$. If $\langle\alpha, \mathbf{x}\rangle>m$ for all $\mathbf{x} \in R$ we deduce that $H_{\alpha, m}$ separates $R$ from the origin, which does not necessarily imply that $H_{\alpha, m}$ supports (i.e. is a separating wall of) $R$. However, the above implication is true in the special case where $\alpha$ is a simple root. To see this, notice that when $\alpha$ is a simple root there exists no 
pair $\beta, \gamma$ of positive roots with $\alpha=\beta+\gamma$. Thus, there can be no inequalities of the form $\langle\beta, \mathbf{x}\rangle>\ell,\langle\gamma, \mathbf{x}\rangle>m$ holding for all $\mathbf{x} \in R$, implying that $\langle\alpha, \mathbf{x}\rangle>m$ for all $\mathbf{x} \in R$. We therefore conclude that, if $\alpha$ is a simple root, then the hyperplane $H_{\alpha, m}$ is a separating wall of $R \in \mathcal{R}_{n}^{m}$ if and only if $\langle\alpha, \mathbf{x}\rangle>m$ for all $\mathbf{x} \in R$.

Consider now a partition $\Lambda=\left(\lambda_{1}, \lambda_{2}, \ldots, \lambda_{n}\right) \in \mathcal{P}_{n}^{m}$ with $\lambda_{i}=m(n-i+1)$. From Theorem 3.1 we have that $\psi^{-1}$ sends $\Lambda$ to a region $R \in \mathcal{R}_{n}^{m}$ with $\sum_{\ell=i}^{n} k_{i, \ell}=\lambda_{i}=$ $m(n-i+1)$. Since all the entries of the tableau are positive and less than or equal to $m$, the above equality leaves no other choice but $k_{i, \ell}=m$ for all $i \leqslant \ell \leqslant n$. Thus $k_{i, i}=m$, or equivalently, $\left\langle\alpha_{i}, \mathbf{x}\right\rangle>m$ for all $\mathbf{x} \in R$, which in view of our earlier remark, means that $H_{\alpha_{i}, m}$ is a separating wall of $R$.

Corollary 3.3. The map $\varphi: \mathcal{R}_{n}^{m} \rightarrow \mathcal{P}_{n}^{m}$ of Theorem 3.1 restricts to a bijection between bounded regions in $\mathcal{R}_{n}^{m}$ and partitions in $\mathcal{P}_{n}^{m}$ that never attain their maximal size, i.e $\lambda_{i}<m(n-i+1)$ for every $i \in[n]$.

Proof. As we mentioned in the introduction, a dominant region $R$ is bounded if and only if $0<\left\langle\mathbf{x}, \alpha_{i}\right\rangle<m$ for all $\mathbf{x} \in R$ and all simple roots $\alpha_{i}, i \in[n]$. In view of Definition 2.1, such a region will be represented by a Shi tableau for which $k_{i, i} \neq m$ for every $i \in[n]$. Clearly, this happens if and only if $\lambda_{i} \neq m(n-i+1)$ and this completes our proof.

Example 3.4. The following figure shows how, given a partition $\Lambda \in \mathcal{P}_{n}^{m}$, we define the entries of the corresponding Shi tableau $\varphi^{-1}(\Lambda)$. It is immediate from (3.1) that the first entry to be defined is $k_{n, n}:=\lambda_{n}$ and each other $k_{i, j}$ is defined recursively from the entries of the tableau lying below and to its left.

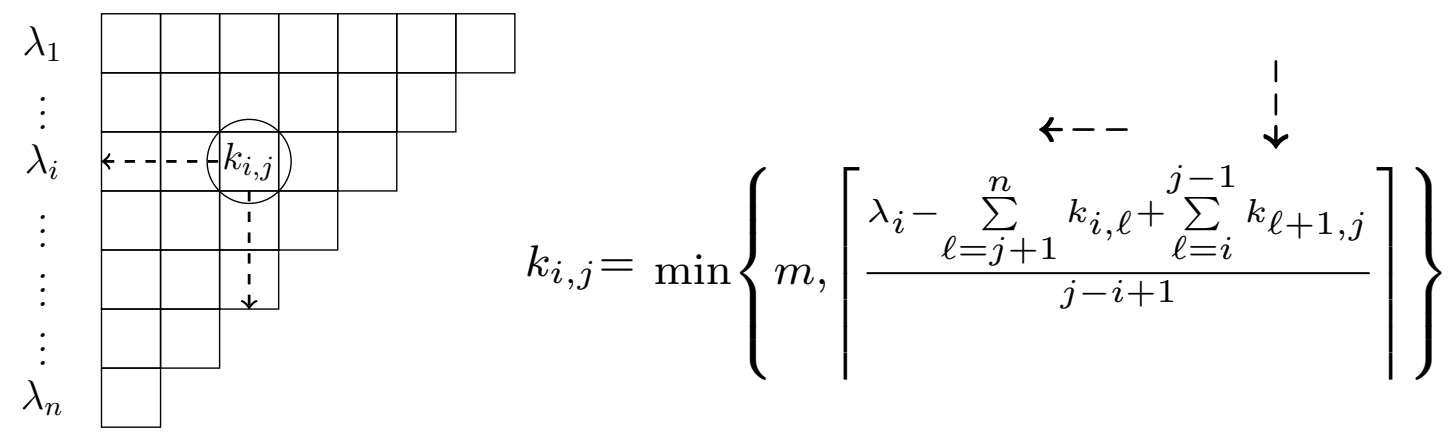

Example 3.5. For $\Phi=A_{4}, m=3$ and $\Lambda=(10,3,2,0)$, the tableau $\varphi^{-1}(\Lambda)$ is constructed as shown in Figure 5.

\section{From facets of $\mathcal{D}_{n}^{m}$ to partitions in $\mathcal{P}_{n}^{m}$}

In this section we present Bijection 3 (Theorem 4.4). We first describe a natural bijection between the set of facets $\mathcal{D}_{n}^{m}$ and that of partitions $\mathcal{P}_{n}^{m}$ (Theorem 4.2) which motivated ours and we explain why it cannot be adapted in our case. In order to describe our bijections, we need the following definition. 


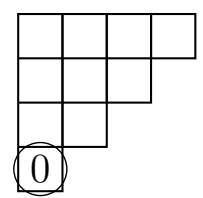

$k_{4,4}=0$

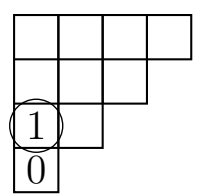

$k_{3,4}=$

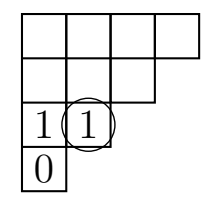

$k_{3,3}=$

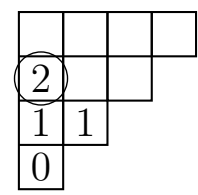

$k_{2,4}=$

$$
\min \left\{3,\left\lceil\frac{2+0}{4-3+1}\right\rceil\right\} \quad \min \left\{3,\left\lceil\frac{2-1}{3-3+1}\right\rceil\right\} \quad \min \left\{3,\left\lceil\frac{3+1+0}{4-2+1}\right\rceil\right\}
$$

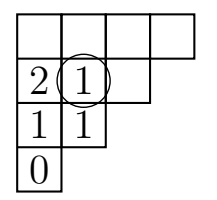

$k_{2,3}=$

$$
\min \left\{3,\left\lceil\frac{3-2+1}{3-2+1}\right\rceil\right\}
$$
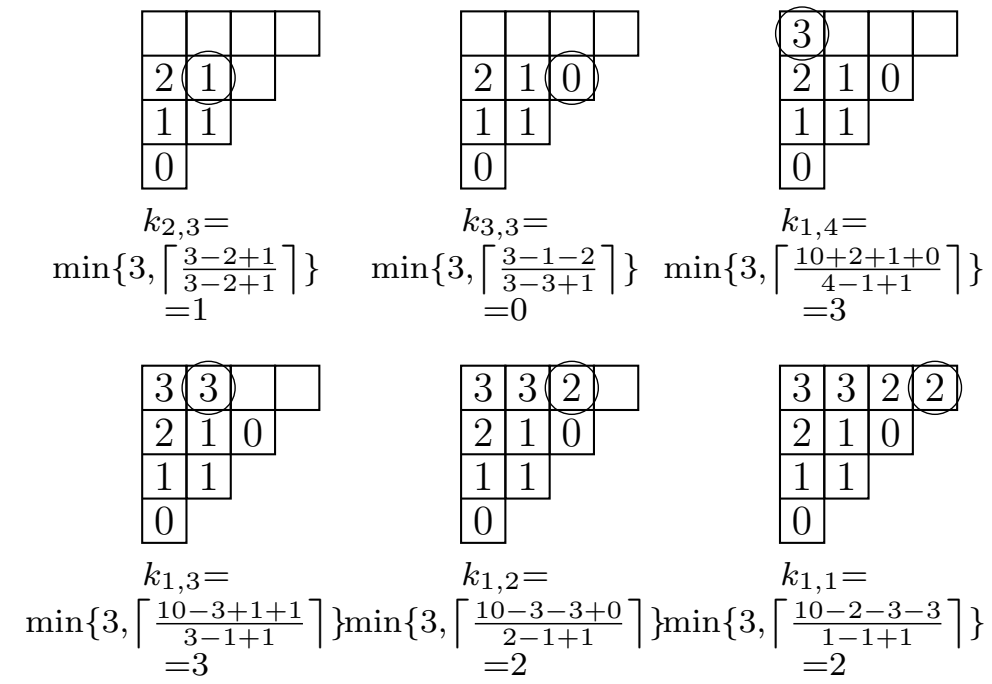

$k_{1,3}=$

$\min \left\{3,\left\lceil\frac{3-1-2}{3-3+1}\right\rceil\right.$
$=0$

\} $\min \left\{3,\left\lceil\frac{10+2+1+0}{4-1+1}\right\rceil\right\}$

$=3$

Figure 5:

Definition 4.1. Let $P$ be a polygon with $m(n+1)+2$ vertices labeled by the distinct integers in the set $[m(n+1)+1]_{0}$. Each diagonal $d$ of $P$ has two labeled endpoints. The smaller (respectively larger) among these two labels is called the initial (repsectively terminal) point of $d$.

For example Figure 6 shows a dissection whose diagonals are oriented from their initial to their terminal endpoint.

Without going into further details, we note that the following theorem is a restatement of [19, Theorem 1] in the special case where all epsilons are equal to 1 and the base region is the $(m+2)$-gon containing 0 . Alternately, it can be derived from results in [15], or be proved using arguments similar to those employed in the proof of Theorem 4.4

Theorem 4.2. Let $P$ be an $m(n+1)+2$-gon with vertices labeled by the integers in $[m(n+1)+1]_{0}$ in counterclockwise order. Let $D$ be an $(m+2)$-angulation of $P$ with initial points $\left\{t_{1}, t_{2}, \ldots, t_{n}\right\}$, where we assume that $t_{1} \geqslant t_{2} \geqslant \cdots \geqslant t_{n}$. The map $\psi^{\prime}: \mathcal{D}_{n}^{m} \rightarrow \mathcal{P}_{n}^{m}$ defined by letting $\psi^{\prime}(D)=\left(t_{1}, t_{2}, \ldots, t_{n}\right)$, for every $D \in \mathcal{D}_{n}^{m}$, is a bijection.

Even though the map $\psi^{\prime}$ describes a simple and natural way to associate integer partitions to polygon dissections, it can not be adopted in our case since the composition $\varphi^{-1} \circ \psi^{\prime}$ does not preserve the property of Bijection 1 . Indeed, let $P$ be an $(m(n+$ 
$1)+2$ )-gon with vertices labeled as in Theorem 4.2 (i.e. counterclockwise) and $D$ be the dissection with diagonals $\{i m, m(n+1)+1\}, i \in[n]$. These diagonals have initial points $\{m, 2 m, \ldots, m n\}$ and thus $\psi^{\prime}(D)=(m n, m(n-1), \ldots, m)$. When $n \geqslant 2$, the diagonals in $D$ are all incident to the vertex $m(n+1)+1$ and hence do not form a "snake" (see Figure 6 bottom dissection). On the other hand, from Theorem 3.2 we have that $\varphi^{-1}\left(\psi^{\prime}(D)\right)=\varphi^{-1}((m n, m(n-1), \ldots, m))$ is the Shi tableau with all entries equal to $m$. This tableau represents the region whose separating walls are all the hyperplanes $H_{\alpha_{i}, m}$ with $i \in[n]$. The property in Bijection 1 requires that the above mentioned region should be mapped to the facet $\left\{-\alpha_{i}, i \in[n]\right\}$ of the cluster complex, i.e. the snake. Since the diagonals in $D$ do not form a "snake", $\psi^{\prime}$ is not the right map.

Although the map $\psi^{\prime}$ does not give us the required characterization, it constitutes the key-idea towards the construction of Bijection 3. More precisely, in Theorem 4.4 we define a map which (again) sends each dissection to the set of its initial points, but this time the vertices of $P$ are labeled in such a way that eventually the property of Bijection 1 is preserved. We remark that since the definition of the negative simpe roots depends on the labeling of the vertices (see Section 2.2), a relabeling of the vertices will force us to redescribe the negative simple roots as well. We describe in detail the new labeling, which we call alternating labeling and the way the negative simple roots are defined with respect to it, in the next paragraph.

\subsection{Alternating labeling and negative simple roots.}

In the remainder of the paper, when we write that a vertex of a polygon $P$ lies on the right (resp. on the left) of some other vertex of $P$, we mean on the right (resp. on the left) with respect to the center of the polygon.

Definition 4.3. Let $P$ be a polygon with $m(n+1)+2$ vertices labeled from 0 to $m(n+1)+1$ as follows: Fix a vertex labeled with 0 . The vertices on the right of 0 are labeled with the numbers $k \in[m(m+1)+1]$ for which the integer $\left\lfloor\frac{k}{m}\right\rfloor$ is even and so that the labels increase in the counterclockwise direction from vertex 0 . Similarly, the vertices on the left of 0 are labeled with those $k \in[m(m+1)+1]$ for which the integer $\left\lfloor\frac{k}{m}\right\rfloor$ is odd and so that the labels increase in the clockwise direction. We call this the alternating labeling of $P$. (See Figure $7(\alpha)$ ).

Let $P$ be an $(m(n+1)+2)$-gon, fix some vertex 0 and consider its alternating labeling. By the definition of the alternating labeling, one can see that the diagonals with endpoints $m(n-i+1), m(n-i+2)$, where $i \in[n]$, form a "snake" (see Figure $7(\alpha))$. Thus, for each $i \in[n]$, we identify the negative simple root $-\alpha_{i}$ with the diagonal having endpoints $m(n-i+1), m(n-i+2)$. Under this identification, the set of colored positive roots can be defined as in Section 2.2.

In what follows, we say that a vertex $u \in P$ lies $(k+1)$-many vertices apart from another vertex $v \in P$, if there exist exactly $k$ consecutive vertices between $u$ and $v$. For example, in Figure $7(\beta)$ both 3 and 14 lie four vertices apart from 10. Now we are ready to state and prove the main theorem of this section. 

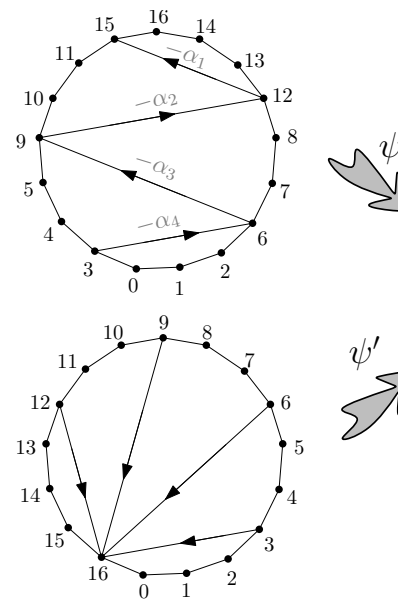

$\Lambda=(12,9,6,3)$

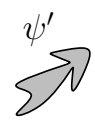

\begin{tabular}{|l|l|l|l|}
\hline 3 & 3 & 3 & 3 \\
\hline 3 & 3 & 3 & \multicolumn{1}{|}{} \\
\cline { 1 - 2 } 3 & 3 & \multicolumn{2}{|}{} \\
\cline { 1 - 2 } 3 & \multicolumn{2}{|c}{} \\
\cline { 1 - 1 } & &
\end{tabular}

Figure 6: The Shi tableau $T$ with all entries equal to 3 corresponds to the unique dominant region $R$ in $\operatorname{Cat}^{3}\left(A_{4}\right)$ having separating walls the hyperplanes $H_{\alpha_{1}, 3}, H_{\alpha_{2}, 3}, H_{\alpha_{3}, 3}, H_{\alpha_{4}, 3}$. Bijection 1 requires that $R$ should be mapped to the facet $D=\left\{-\alpha_{1},-\alpha_{2},-\alpha_{3},-\alpha_{4}\right\}$ of $\Delta^{3}\left(A_{2}\right)$, i.e the dissection containing the "snake". This happens in the top dissection (alternating labeling) and fails in the bottom one (counterclockwise labeling).

Theorem 4.4. Let $P$ be an $(m(n+1)+2)$-gon with vertices labeled as in Definition 4.3. Let $D \in \mathcal{D}_{n}^{m}$ be a dissection with initial points $\left\{t_{1}, t_{2}, \ldots, t_{n}\right\}$, where we assume that $t_{1} \geqslant t_{2} \geqslant \cdots \geqslant t_{n}$. The map $\psi: \mathcal{D}_{n}^{m} \rightarrow \mathcal{P}_{n}^{m}$ defined by letting

$$
\psi(D)=\left(t_{1}, t_{2}, \ldots, t_{n}\right)
$$

for every $D \in \mathcal{D}_{n}^{m}$, is a bijection with the property that $D$ contains the diagonal corresponding to the negative simple root $-\alpha_{i}$ if and only if $t_{i}=m(n-i+1)$.

Proof. We first need to prove that $\psi$ is well defined, i.e. that $t_{i} \leqslant m(n-i+1)$ for every $i \in[n]$. Assume on the contrary that there exists at least one $i \in[n]$ such that $t_{i}>m(n-i+1)$ and let $i_{0} \in[n]$ be the greatest such index. This means that $t_{i} \leqslant$ $m(n-i+1)$ for all $i_{0}<i \leqslant n$ and $t_{i_{0}}>m\left(n-i_{0}+1\right)$. Next notice that, by the definition of the alternating labeling, the diagonal $-\alpha_{i_{0}}$ dissects $P$ into an $\left(m i_{0}+2\right)$-gon $P^{\prime}$ and an $\left(m\left(n-i_{0}+1\right)+2\right)$-gon $P^{\prime \prime}$ with non common vertices $\mathcal{V}^{\prime}=\left\{m\left(n-i_{0}+1\right)+1, m\left(n-i_{0}+1\right)+\right.$ $2, \ldots, m(n+1)+1\} \backslash\left\{m\left(n-i_{0}+2\right)\right\}$ and $\mathcal{V}^{\prime \prime}=\left\{0,1, \ldots, m\left(n-i_{0}+1\right)-1, m\left(n-i_{0}+1\right)\right\}$ respectively (see Figure 8 ).

Since $m\left(n-i_{0}+1\right)<t_{i_{0}} \leqslant t_{i_{0}-1} \leqslant \cdots \leqslant t_{1}$ we deduce that the $m$-diagonals $d_{1}, d_{2}, \ldots, d_{i_{0}}$ with initial points $t_{1}, \ldots, t_{i_{0}}$ should have both their endpoints in $\mathcal{V}^{\prime}$. Indeed, any diagonal with each endpoint in one of $\mathcal{V}^{\prime}$ and $\mathcal{V}^{\prime \prime}$ has initial point in $\mathcal{V}^{\prime \prime}$ (since the vertex labels in $\mathcal{V}^{\prime \prime}$ are smaller). We thus conclude that the $\left(m i_{0}+2\right)$-gon $P^{\prime}$ has $i_{0}$ many $m$-diagonals, which is a contradiction (it cannot have more than $i_{0}-1$ ). So the map $\psi$ is well defined.

To see that $\psi$ is a bijection we construct its inverse. We set $\psi=\psi_{n}$ and proceed by induction on $n$, the case $n=1$ being trivial. Assume that we have constructed the 


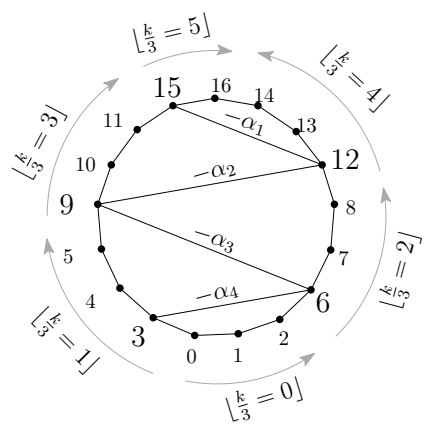

$(\alpha)$

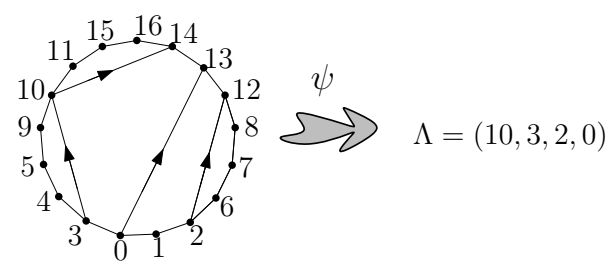

$(\beta)$

Figure 7: $(\alpha)$ The alternating labeling of a 17-gon for $m=3$ and $n=4$. The negative simple roots $-\alpha_{1},-\alpha_{2},-\alpha_{3},-\alpha_{4}$ correspond to the diagonals $\{12,15\},\{9,12\},\{6,9\},\{3,6\}$ respectively. $(\beta)$ An example of the map $\psi$ of Theorem 4.4.

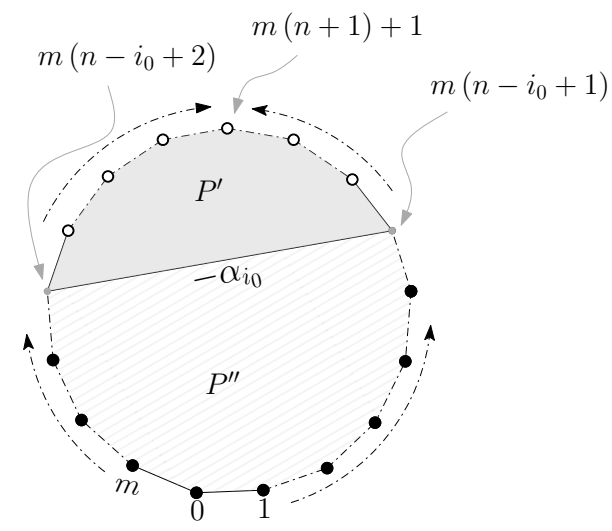

Figure 8: The vertices in $\mathcal{V}^{\prime}$ and $\mathcal{V}^{\prime \prime}$ are the circles and black disks respectively. The circular arrows show the direction in which the labeling of the vertices increases from 0 to $m(n+1)+1$.

bijection for $n-1$. Let $\left(\lambda_{1}, \lambda_{2}, \ldots, \lambda_{n}\right)$ be an element of $\mathcal{P}_{n}^{m}$ and $P$ be an $(m(n+1)+2)$ gon with the alternating labeling. We will construct a dissection $D \in \mathcal{D}_{n}^{m}$ of $P$ with set of initial points $\left\{\lambda_{1}, \lambda_{2}, \ldots, \lambda_{n}\right\}$. To this end, consider the vertex of $P$ labeled by $\lambda_{1}$. Let $\bar{\lambda}_{1}$ and $\tilde{\lambda}_{1}$ be the two vertices which lie $(m+1)$-many vertices apart from $\lambda_{1}$ and without loss of generality assume that $\bar{\lambda}_{1} \geqslant \tilde{\lambda}_{1}$ (equality holding only when $n=1$ ). We claim that $\bar{\lambda}_{1}>\lambda_{1}$. To prove our claim we need to separate two cases:

(i) $m(n-1) \leqslant \lambda_{1} \leqslant m n$ (Figure $9(\alpha)$ ). In this case $\lambda_{1}$ is one of the vertices (except for $m(n+1))$ of the $(m+2)$-gon $P^{\prime}$ defined by $-\alpha_{1},-\alpha_{2}$. It is then not hard to see that $\bar{\lambda}_{1}$ should lie in the $(m+2)$-gon $P^{\prime \prime}$ defined by $-\alpha_{1}$ and the boundary of $P$. Since the vertex labels in $P^{\prime \prime}$ are $\{m n+1, \ldots, m(n+1)+1\}$, our claim holds.

(ii) $\lambda_{1}<m(n-1)$ (Figure $\left.9(\beta)\right)$. In this case we can assume that $\kappa m \leqslant \lambda_{1}<(\kappa+1) m$ for some $0 \leqslant \kappa \leqslant n-2$. Pictorially, this means that $\lambda_{1}$ is one of the $m$-many contiguous 


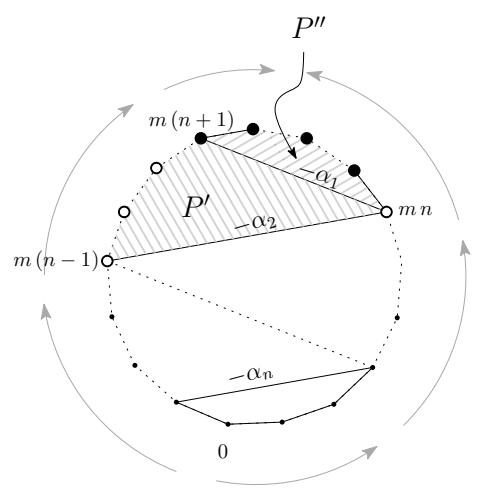

$(\alpha)$

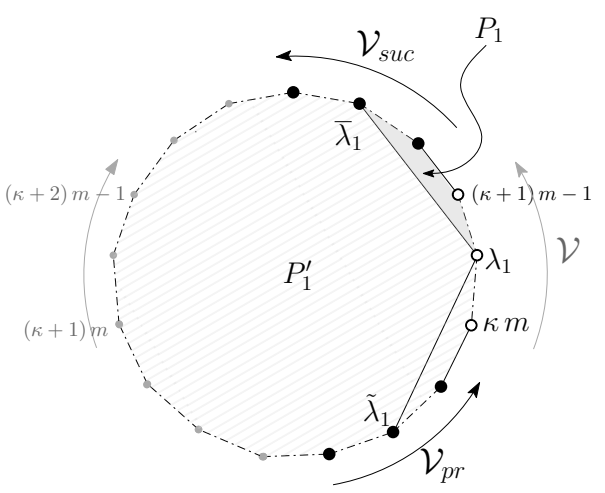

$(\beta)$

Figure 9: Illustration of the two cases for the claim in the proof of $\psi^{-1}$. The circular arrows show the direction in which the labeling of the vertices in- creases from 0 to $m(n+1)+1$.

vertices in $\mathcal{V}=\{\kappa m, \kappa m+1, \ldots,(\kappa+1) m-1\}$ (See Figure $8(\beta))$. Clearly, none of $\bar{\lambda}_{1}, \tilde{\lambda}_{1}$ is in $\mathcal{V}$ since they both lie $(m+1)$-many vertices apart from $\lambda_{1}$. Thus, $\bar{\lambda}_{1}, \tilde{\lambda}_{1}$ belong to one and not the same of $\mathcal{V}_{p r}$ and $\mathcal{V}_{\text {suc }}$, where $\mathcal{V}_{p r}, \mathcal{V}_{\text {suc }}$ is the set of the $m$ vertices preceding and succeeding (in the labeling order) respectively those in $\mathcal{V}$. From the definition of the alternating labeling we deduce that $\mathcal{V}_{\text {suc }}=\{(\kappa+2) m, \kappa m+1, \ldots,(\kappa+3) m-1\}$. Since we have assumed that $\bar{\lambda}_{1} \geqslant \tilde{\lambda}_{1}$, we deduce that $\bar{\lambda}_{1} \in \mathcal{V}_{\text {suc }}$. This, combined with our assumption that $\lambda_{1}<(\kappa+1) m$, implies that indeed $\bar{\lambda}_{1}>\lambda_{1}$.

We set $d_{1}$ to be the diagonal of $P$ with endpoints $\lambda_{1}$ and $\bar{\lambda}_{1}$. Clearly $d_{1}$ dissects $P$ into an $(m+2)$-gon and an $(m n+2)$-gon, which we denote by $P_{1}$ and $P_{1}^{\prime}$ respectively. Arguing as in the proof of our earlier claim, one can see that not only $\bar{\lambda}_{1}>\lambda_{1}$, but also $\lambda_{1}$ is the smallest vertex label in $P_{1}$. Thus, since $\left\{\right.$ vertex labels in $\left.P_{1}\right\}>\lambda_{1} \geqslant \lambda_{2} \geqslant \cdots \geqslant \lambda_{n}$, we deduce that $\lambda_{2}, \ldots, \lambda_{n}$ are vertex labels in $P_{1}^{\prime}$. From the induction hypothesis, there exists a dissection of the $(m n+2)$-gon $P_{1}^{\prime}$ having $m$-diagonals $d_{2}, d_{3}, \ldots, d_{n}$ with initial points $\lambda_{2}, \lambda_{3}, \ldots, \lambda_{n}$ respectively. We leave it to the reader to check that the map which sends $\lambda$ to the dissection containing the diagonals $d_{1}, d_{2}, \ldots, d_{n}$ is indeed the inverse of $\psi$. For an example of the map $\psi^{-1}$ we refer the reader to Figure 10.

To prove that $\psi$ has the property in the statement of the theorem, we refer to Figure 8 where we set $i_{0}:=i$. Let $D$ be a dissection in $\mathcal{D}_{n}^{m}$ having negative simple root $-\alpha_{i}$. By the representation of the negative simple roots as a "snake", it follows that $D$ contains the diagonal $\{m(n-i+1), m(n-i+2)\}$ which dissects $P$ into an $(m i+2)$-gon $P^{\prime}$ and an $(m(n-i+1)+2)$-gon $P^{\prime \prime}$. Now, since $D$ is an $(m+2)$-angulation of $P$ containing $n+1$ non-crossing diagonals, one of which is $-\alpha_{i}$, we are forced to have $i-1$ diagonals with both endpoints in $P^{\prime}$ and $n-i+1$ diagonals with both endpoints in $P^{\prime \prime}$. From Figure 8 one can see that each diagonal in $P^{\prime \prime}$ has initial point with label strictly smaller than $m(n-i+1)$ and each diagonal in $P^{\prime}$ has initial point with label $\geqslant m(n-i+1)$. From this, we conclude that the diagonal $-\alpha_{i}$ is the $i$-th one, as we order them decreasingly according to their initial points. This means that $\lambda_{i}=m(n-i+1)$. 


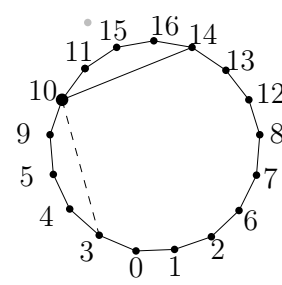

$(\alpha)$

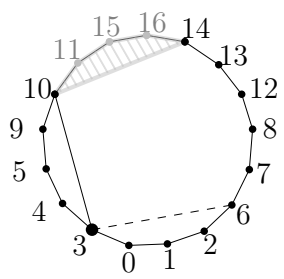

$(\beta)$

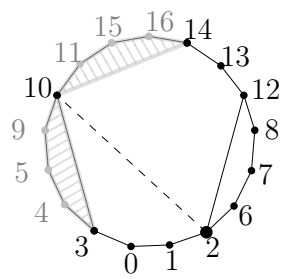

$(\gamma)$

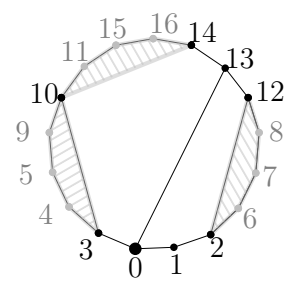

$(\delta)$

$$
\Lambda=(10,3,2,0) \text { and } m=3
$$
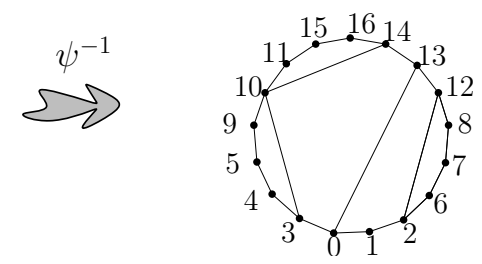

Figure 10: Example of the construction of the facet $\psi^{-1}(\Lambda)$ when $\Lambda=(10,3,2,0) \in \mathcal{P}_{4}^{3}$ : $(\alpha)$ among the two vertices lying $(m+1=4)$-many vertices apart from the vertex 10 , we choose the one with the greater label i.e. 14 and cut away the the $(m+2)$-polygon (5-gon) defined by the diagonal $\{10,14\}$.

$(\beta)$ among the two vertices lying four vertices apart from the vertex 3 , we choose the one with the greater label i.e. 10 and cut away the 5 -gon defined by the diagonal $\{3,10\}$.

$(\gamma)$ among the two vertices lying four vertices apart from the vertex 2 , we choose the one with the greater label i.e. 12 and we cut away the 5-gon defined by the diagonal $\{2,10\}$. $(\delta)$ there is only one vertex which is four vertices apart from 0 , namely the one labeled by 13 . We draw the unique diagonal $\{0,13\}$.

To prove the reverse, let $\Lambda=\left(\lambda_{1}, \ldots, \lambda_{n}\right) \in \mathcal{P}_{n}^{m}$ with $\lambda_{i}=m(n-i+1)$. By the definition of $\psi$, the dissection $D=\psi^{-1}(\Lambda)$ has diagonals $d_{1}, \ldots, d_{i}, \ldots, d_{n}$ with initial points $\lambda_{1} \geqslant \ldots \geqslant \lambda_{i} \geqslant \ldots \geqslant \lambda_{n}$ respectively. We define $P^{\prime}$ and $P^{\prime \prime}$ as before and we notice that the diagonals $d_{1}, \ldots, d_{i}$ should have both their endpoints in $P^{\prime}$, since otherwise their initial points would have labels strictly less than $m(n-i+1)$. Since we cannot have $i$ diagonals in an $(m i+2)$-gon, we are forced to have $d_{i}:=-\alpha_{i}$.

Recalling that $\Delta_{+}^{m}(\Phi)$ is the subcomplex of $\Delta^{m}(\Phi)$ having faces which do not contain any negative simple root of $\Phi$, one immediately derives the following:

Corollary 4.5. The map $\psi: \mathcal{R}_{n}^{m} \rightarrow \mathcal{P}_{n}^{m}$ of Theorem 4.4 restricts to a bijection between the set of facets of the positive cluster complex $\Delta_{+}^{m}\left(A_{n}\right)$ and partitions in $\mathcal{P}_{n}^{m}$ which never attain their maximal size.

\section{Conclusion and ongoing work}

In this paper we present a bijection between the set of dominant regions in the $m$-Catalan arrangement $\operatorname{Cat}^{m}\left(A_{n}\right)$ and that of facets of the $m$-generalized cluster complex $\Delta^{m}\left(A_{n}\right)$, 
where $m \in \mathbb{N}$. Our bijection restricts to one between regions in $\operatorname{Cat}^{m}\left(A_{n}\right)$ having simple separating wall the hyperplane $H_{\alpha_{i}, m}$ and facets of $\Delta^{m}\left(A_{n}\right)$ with negative simple root $-\alpha_{i}$. We achieve our goal by using, as intermediate step, integer partitions which fit in an $m$-dilated staircase of size $n$.

\subsection{Ongoing work}

In our future work [8], we focus on types $B_{n}$ and $C_{n}$. More precisely, we use the realization of facets of the generalized cluster complex $\Delta^{m}\left(B_{n}\right)$ (or $\Delta^{m}\left(C_{n}\right)$ ) as centrally symmetric $(m+2)$-angulations of a $(2 m n+2)$-gon [10, Section 5.2]. Using central symmetry and Theorem 4.4 we are able to biject facets of $\Delta^{m}\left(B_{n}\right)$ (or $\left.\Delta^{m}\left(C_{n}\right)\right)$ to the set $\mathcal{P}^{m}\left(B_{n}\right)$ of partitions $\left(\lambda_{1}, \lambda_{2}, \ldots, \lambda_{n}\right)$ with $0 \leqslant \lambda_{i} \leqslant m n$. Using a bijection from [18] which sends partitions in $\mathcal{P}^{m}\left(B_{n}\right)$ to pairs of an integer $k \in[n m]_{0}$ and partitions in $\mathcal{P}_{n-1}^{m}$, we can further characterize facets of $\Delta^{m}\left(B_{n}\right)$ (or $\Delta^{m}\left(C_{n}\right)$ ) containing some negative simple root, in terms of the part-sizes of the corresponding partition. However, the type- $B$ (or $C$ ) analogue of Bijection 2, which associates partitions to regions, turns out to be much more difficult and still remains elusive.

\subsection{Open problem}

Combining results from [4] and [10] it is not hard to prove the following:

Proposition 5.1. Let $\Phi$ be a finite (possibly reducible) crystallographic root system and let $\left\{\alpha_{1}, \alpha_{2}, \ldots, \alpha_{n}\right\}$ be its set of simple roots. For any $J \subseteq[n]$, the number of facets of $\Delta^{m}(\Phi)$ containing exactly the negative simple roots $-\alpha_{i}$ with $i \in J$, is equal to the number of dominant regions in $\mathrm{Cat}^{m}(\Phi)$ with simple separating walls $H_{\alpha_{i}, m}$ with $i \in J$.

When $\Phi=A_{n}$, the property of Bijection 1 agrees with the above proposition. It is therefore natural to seek a generalization of Bijection 1 for any finite crystallographic root system $\Phi$. In other words, it would be interesting to find a uniform bijection from the set of facets of $\Delta^{m}(\Phi)$ to the set dominant regions in $\mathrm{Cat}^{m}(\Phi)$ that agrees with Proposition 5.1.

\section{Acknowledments}

We are grateful to Philippe Nadeau for helpful discussions and Allesandro Conflitti for providing us with Formula (3.1). We would also like to thank the anonymous referee for the careful reading and for many valuable comments on the presentation of the paper.

\section{References}

[1] D. Armstrong, C. Stump, and H. Thomas, A uniform bijection between nonnesting and noncrossing partitions, Trans. Amer. Math. Soc. 365 (2013), no. 8, 4121-4151.

[2] C.A. Athanasiadis, Generalized Catalan numbers, Weyl groups and arrangements of hyperplanes, Bull. London Math. Soc. 36 (2004), 294-302. 
[3] On a refinement of the generalized Catalan numbers for Weyl groups, Trans. Amer. Math. Soc. 357 (2005), 179-196.

[4] C.A. Athanasiadis and E. Tzanaki, On the enumaration of positive cells in generalized cluster complexes and Catalan hyperplane arrangements, J. Algebraic Comin. 23 (2006), no. 4, 355-375.

[5] Mireille Bousquet-Mélou, Éric Fusy, and Louis-François Préville-Ratelle, The number of intervals in the m-Tamari lattices, Electron. J. Combin. 18 (2011), no. 2, Paper $31,26 \mathrm{pp}$.

[6] A.B. Buan, I. Reiten, and H. Thomas, m-noncrossing partitions and $m$-clusters, 21st International Conference on Formal Power Series and Algebraic Combinatorics (FPSAC 2009), 2009, pp. 145-154.

[7] A. Conflitti and R. Mamede, On Noncrossing and Nonnesting partitions of Type D, Ann. Comb. 15 (2011), 637-654.

[8] S. Fishel, M. Kallipoliti, and E. Tzanaki, Integer partition models for generalized cluster complexes and extended Catalan arrangements. In preparation.

[9] S. Fishel, E. Tzanaki, and M. Vazirani, Counting Shi regions with a fixed separating wall, Annals of Combinatorics (2012). To appear.

[10] S. Fomin and N. Reading, Generalized cluster complexes and Coxeter combinatorics, Int. Math. Res. Not. 44 (2005), 2709-2757.

[11] S. Fomin and A. Zelevinsky, Cluster algebras I: Foundations, J. Amer. Math. Soc. 15 (2002), 497-529.

[12] _ Y-systems and generalized associahedra, Ann. of Math. 158 (2003), no. 2, 997-1018.

[13] I. P. Goulden and Luis G. Serrano, Maintaining the spirit of the reflection principle when the boundary has arbitrary integer slope, J. Combin. Theory Ser. A 104 (2003), no. 2, 317-326.

[14] J. Haglund, J. Morse, and M. Zabrocki, A compositional shuffle conjecture specifying touch points of the Dyck path, Canad. J. Math. 64 (2012), no. 4, 822-844.

[15] P. Hilton and J. Pedersen, Catalan numbers, their generalization and their uses, Math. Intell. 13 (1991), 64-75.

[16] Christian Krattenthaler, The F-triangle of the generalised cluster complex, Topics in discrete mathematics, 2006, pp. 93-126.

[17] R. Mamede, A bijection between noncrossing and nonnesting partitions of type $A, B$ and $C$, Contrib. Discrete Math. 6 (2011), no. 2, 70-90.

[18] S. G. Mohanty, Lattice path counting and applications, Academic Press [Harcourt Brace Jovanovich Publishers], New York, 1979. Probability and Mathematical Statistics. 
[19] J. Ç. Przytycki and A. S. Sikora, Polygon Dissections and Euler, Fuss, Kirkman, and Cayley Numbers, J. Combin. Theory Ser. A 92 (2000), 68-76.

[20] N. Reading, Clusters, Coxeter-sortable elements and noncrossing partitions, Trans. Amer. Math. Soc. 359 (2007), 5931-5958.

[21] M. J. Richards, Some decomposition numbers for Hecke algebras of general linear groups 119 (1996), no. 3, 383-402.

[22] J-Y. Shi, Alcoves corresponding to an affive Weyl group, J. London Math. Soc. 35 (1987), 42-55.

[23] - The number of $\oplus$-sign types, Quart. J. Math. Oxford 48 (1997), 93-105.

[24] Jian Yi Shi, The Kazhdan-Lusztig cells in certain affine Weyl groups, Lecture Notes in Mathematics, vol. 1179, Springer-Verlag, Berlin, 1986.

[25] Richard P. Stanley, Hyperplane arrangements, interval orders, and trees, Proc. Nat. Acad. Sci. U.S.A. 93 (1996), no. 6, 2620-2625.

[26] _ Hyperplane arrangements, parking functions and tree inversions, Mathematical essays in honor of Gian-Carlo Rota (Cambridge, MA, 1996), 1998, pp. 359-375.

[27] E. Tzanaki, Faces of generalized cluster complexes and noncrossing partitions, SIAM J. Discrete Math. 22 (2008), no. 1, 15-30. 